\title{
Incidental lowering of otitis-media complaints in otitis-prone children during COVID-19 pandemic: not all evil comes to hurt
}

\author{
Sara Torretta ${ }^{1,2}$ (1) Pasquale Capaccio ${ }^{1,3}$ • Ilaria Coro $^{4,5}$ - Samantha Bosis ${ }^{4}$ - Maria Elisabetta Pace ${ }^{4,5}$ - Pietro Bosi ${ }^{4,5}$. \\ Lorenzo Pignataro ${ }^{1,2} \cdot$ Paola Marchisio ${ }^{4,5}$
}

Received: 8 June 2020 / Revised: 9 July 2020 / Accepted: 16 July 2020

(C) Springer-Verlag GmbH Germany, part of Springer Nature 2020, corrected publication 2020

\begin{abstract}
Given COVID-19 pandemic periodic outpatient assessment of otitis-prone children regularly followed at our tertiary outpatient clinic of upper respiratory tract infections was discontinued since 9 March. In order to avoid leaving the patients to themselves just during the winter months, which are the most critical ones for these children, we kept in touch with the families of 102 children (mean age $41.4 \pm 14.0$ months) who had had a follow-up visit scheduled during the lockdown, and compensated with telemedicine assessment. This incidentally leads to the unexpected but not at all negative finding that a consistent clinical improvement had been occurred in most $(82.3 \%)$ of children. A statistically significant reduction in the mean number of documented acute otitis media episodes, otorrhea episodes, and systemic antibiotic treatments during the February-April 2020 period compared with February-April 2019 was attested. Clinical evaluation performed in $27.4 \%$ cases revealed normal middle ear findings in all but three (89.3\%) children.

Conclusion: Our data document a global improvement of otitis-prone children in Milan during the Italian lockdown, as a fortuitous and incidental positive effect of the national lockdown.

What is Know:

- During COVID-19 pandemic in Italy any non-urgent medical activity including periodic outpatient assessment of otitis-prone children was discontinued.

- Otitis-prone children experience acute infectious exacerbations mainly in winter.

What is New:

- Most of children reached by means of a telemedicine assessment during lockdown experienced a subjective clinical improvement; clinical assessment at the end of the lockdown revealed normal otoscopic findings in most cases.

- Exceptional circumstances during COVID-19 pandemic had a fortuitous positive effect on otitis-prone children's clinical conditions.
\end{abstract}

Keywords Otitis media $\cdot$ Children $\cdot$ Otitis-prone $\cdot$ COVID-19 $\cdot$ Infection

\section{Abbreviations}

COVID-19 Coronavirus 2019 disease

OPC Otitis-prone children

Communicated by Peter de Winter

Sara Torretta

sara.torretta@unimi.it

1 Department of Otolaryngology and Head and Neck Surgery, Fondazione IRCCS Ca' Granda Ospedale Maggiore Policlinico, Milan, Italy

2 Department of Clinical Sciences and Community Health, University of Milan, Milan, Italy
RAOM Recurrent acute otitis media

CURTI Outpatient clinic of upper respiratory tract infections
3 Department of Biomedical Surgical Dental Science, University of Milan, Milan, Italy

4 Pediatric Highly Intensive Care Unit, Fondazione IRCCS Ca' Granda Ospedale Maggiore Policlinico, Milan, Italy

5 Department of Pathophysiology and Transplantation, University of Milan, Milan, Italy 
AOMsTMP Acute otitis media without spontaneous tympanic membrane perforation.

\section{Introduction}

The stunning diffusion of COVID-19 from our region placed at the epicentre of the Italian epidemic at the end of February 2020 brought us to stop any elective medical activities except for those related to emergency [1]. Periodic outpatient assessment of otitis-prone children (OPC) (i.e. with a history of recurrent acute otitis media-RAOM-defined as $\geq 3$ distinct episodes in a 6 months, or $\geq 4$ in 12 months) [2] who were regularly followed at our tertiary outpatient clinic of upper respiratory tract infections (CURTI) was discontinued since 9 March.

In order to avoid leaving the patients to themselves just during the winter months, which are the most critical ones for OPC, we kept in touch with their families and compensated with telemedicine assessment. This incidentally leads to unexpected but not at all negative findings.

\section{Material and methods}

Family of OPC scheduled for periodic evaluation between 9 March and 17 May were reached by a telephone call performed by resident physicians on 12-19 May. In some children a complete paediatric evaluation with pneumatic otoscopy [3] was executed on 18-19 May. The mean number of episodes of acute otitis media without spontaneous tympanic membrane perforation (AOMsTMP) and otorrhea episodes [4], as well as the number of systemic antibiotic treatments administered in the February-April 2020 period was recorded and compared with the corresponding ones in the February-April 2019 period (data available from children's medical records).

Parents were asked to give a subjective opinion about children's clinical conditions (improved, stable, got worse) during the lockdown. On the basis of information achieved, resident physicians gave indication to perform an urgent visit just at the end of the lockdown, a programmable visit, a visit only in case of needing.

The statistical analysis was mainly designed to detect any possible difference in the number of documented infectious recurrences (number of AOMsTMP and otorrhea episodes and number of systemic antibiotic treatments) between the two periods. The results are given as absolute numbers and percentages, or arithmetical mean values \pm standard deviation. Continuous variables were analysed using the Wilcoxonsigned rank sum test. The data were analysed using STATA 10.0 software (StataCorp, College Station, TX, USA); a $p<$ 0.05 was considered statistically significant.

The protocol was approved by our local Ethics Committee of our hospital and was conducted in accordance with the principles of good clinical practice. Written informed consent was achieved by the children's parents.

\section{Results}

Among 102 children (50\% males; mean age $41.4 \pm 14.0$ months) scheduled for a follow-up visit during the lockdown $30.4 \%$ had a history of RAOM without spontaneous tympanic membrane perforation, and $69.6 \%$ of RAOM with spontaneous tympanic membrane perforations occurring in $\geq$ two thirds of the episodes [4] (Table 1).

Most of parents $(82.3 \%)$ declared that children had improved during the lockdown, and $16.7 \%$ that children were stable; only in one case $(1 \%)$ parents considered clinical conditions of the child getting worse. We documented a statistically significant reduction in the mean number of: episodes of AOMsTMP, otorrhea episodes, and systemic antibiotic treatments during the February-April 2020 period compared with February-April 2019 (Table 1).

Clinical evaluation performed in $27.4 \%$ cases revealed normal middle ear findings in all but three (89.3\%) children; middle ear effusion as previously defined [5] was detected in the remaining children $(10.7 \%)$.

Resident physicians gave indication to non-urgent programmable clinical evaluation in $86.3 \%$ cases and clinical evaluation to be perform only in case of needing in the remaining children $(13.7 \%)$.

\section{Discussion}

Our data document a global improvement of OPC in Milan during the Italian lockdown, attested by a significant reduction in the mean number of episodes of AOMsTMP, otorrhea episodes, and number of antibiotic treatments. This positive trend was confirmed by the parents' subjective judgment, as most of them considered their child clinically improved during the lockdown. Among 28 children who were evaluated just at the end of the lockdown, a complete recovery with normal otoscopic findings was detected in about $90 \%$ of cases. AOM is a widespread and multi-factorial disease, with RAOM mainly affecting males attending day-care, expose to second/third-hand smoke, having older siblings and a positive familiar history of allergy [6-11]. Brief or no breast-feeding, prematurity, the use of pacifier and push-and-pull bottles, air pollution are other predisposing factors [7-11].

Despite temporary restriction in day-care attendance has been proven to partially reduce the number of acute exacerbations $[9,10]$, this alone cannot completely change the natural history of disease.

It is well known that RAOM has a natural tendency to improve with increasing age of the child [12-17]. As a fact, 
Table 1 Demographic and clinical characteristics of the patients and comparison between periods in terms of number of episodes of acute otitis media (AOM) without spontaneous tympanic membrane perforation, otorrhea episodes, and antibiotic treatments (SD: standard deviation)

\begin{tabular}{|c|c|c|c|}
\hline \multicolumn{2}{|l|}{ Characteristics } & \multicolumn{2}{|l|}{ Number $(\%)$} \\
\hline \multicolumn{2}{|l|}{ Mean age (SD), months } & \multicolumn{2}{|l|}{$41.4(14.0)$} \\
\hline \multicolumn{2}{|l|}{ Males } & \multicolumn{2}{|l|}{$51(50.0 \%)$} \\
\hline \multicolumn{2}{|l|}{ Prematurity } & \multicolumn{2}{|l|}{$5(4.9)$} \\
\hline \multicolumn{2}{|l|}{ Low weight at birth } & \multicolumn{2}{|l|}{$3(2.9)$} \\
\hline \multicolumn{2}{|l|}{ Breast-feeding } & \multicolumn{2}{|l|}{$82(80.4)$} \\
\hline \multicolumn{2}{|l|}{ Older siblings } & \multicolumn{2}{|l|}{$54(52.3)$} \\
\hline \multirow[t]{3}{*}{ Order of geniture } & $1 \mathrm{st}$ & \multicolumn{2}{|l|}{$48(47.1)$} \\
\hline & 2 nd & \multicolumn{2}{|l|}{$48(47.1)$} \\
\hline & $3 \mathrm{rd}$ & \multicolumn{2}{|l|}{$6(5.8)$} \\
\hline \multicolumn{2}{|l|}{ Use of pacifier } & \multicolumn{2}{|l|}{$41(40.2)$} \\
\hline \multicolumn{2}{|l|}{ Use of push-and-pull bottle } & \multicolumn{2}{|l|}{$36(37.5)$} \\
\hline \multicolumn{2}{|l|}{ Second/third smoke exposure } & \multicolumn{2}{|l|}{$28(27.7)$} \\
\hline \multicolumn{2}{|l|}{ Familiarity for allergy } & \multicolumn{2}{|l|}{$50(49.5)$} \\
\hline \multicolumn{2}{|l|}{ Increased IgE levels } & \multicolumn{2}{|l|}{$34(38.2)$} \\
\hline \multicolumn{2}{|l|}{ Immunological defect } & \multicolumn{2}{|l|}{$6(6.5)$} \\
\hline \multicolumn{2}{|l|}{ Day-care attendance } & \multicolumn{2}{|l|}{$102(100)$} \\
\hline \multicolumn{2}{|l|}{ Adenoidal disease } & \multicolumn{2}{|l|}{$14(13.7)$} \\
\hline \multicolumn{2}{|l|}{ Hexavalent-vaccination } & $102(100)$ & \\
\hline Anti-pneumococcal vaccination & & $102(100)$ & \\
\hline Flu vaccination (winter 2019-2020) & & $60(60.6)$ & \\
\hline Comparison between periods & February-April 2019 & February-April 2020 & $p$ value \\
\hline Mean no. of AOM episodes/month (SD) & $0.37(0.64)$ & $0.07(0.35)$ & $<0.001$ \\
\hline Mean no. of otorrhea episodes/month (SD) & $0.48(0.80)$ & $0.01(0.09)$ & $<0.001$ \\
\hline Mean no. of antibiotics/month (SD) & $0.85(0.88)$ & $0.09(0.38)$ & $<0.001$ \\
\hline
\end{tabular}

Rosenfeld et al. [13] in their systematic literature review and metanalisis attested that only $17 \%$ of OPC remained classified like this and even that $41 \%$ did not experience any further AOM episode during a median observation period of 6 months (range 10 weeks to 2 years). The authors [13] also reported that the mean baseline rate of $\mathrm{AOM}$ recurrence was at least 0.46 episodes per patient/month with a cumulative recurrence rate of 0.23 (95\% CI 0.18-0.28) AOM episodes per patient/month during the study period.

Even if our study has some limitations (i.e. the retrospective nature and the lack of a control group) mainly due to the fact that it was performed during the Italia lockdown for the COVID-19 pandemic, even considering that the natural history of RAOM tends to spontaneous resolution, the reduction in the mean number of AOM episodes per patient/month here documented appears to be more consistent than that generally reported by literature [12, 13, 15-17].

Under these circumstances, the significant improvement here attested could be considered an incidental positive effect of the national lockdown: exceptional circumstances during COVID-19 pandemic totally modified not only OPC way of living, but subvert social habits at their foundation with confinement of people in their own home. Abolition of any contact of OPC with environment and people other than the ones strictly belonging to the domestic setting, rather than avoidance of the sole inter-personal contacts between children at day-care could be responsible for this result; reduction in air pollution in our metropolitan city during the lockdown could also partially account for it.

Reassessment of the patients during the next winter season would be useful to verify this supposition.

Authors' contributions ST: writing and data analysis;

PC: helping in drafting the paper and revising it;

LP: helping in drafting the paper and revising it;

IC: data collection;

SB: data collection;

MEP: data collection;

PT: data collection;

PM: conception of the work and revising the paper.

\section{Compliance with ethical standards}

Conflict of interest The authors declare that they have no conflict of interest.

Ethical approval The protocol was approved by our local Ethics Committee of our hospital. 


\section{References}

1. Torretta S, Capaccio P, Gaffuri M et al (2020) ENT management of children with adenotonsillar disease during COVID-19 Pandemic. Ready to start again? Int J Ped Otorhinolaryngol. https://doi.org/10. 1016/j.ijporl.2020.110

2. Morris MC, Almudevar AL, Casey JR, Pichichero ME (2015) Familial and microbiological contribution to the otitis-prone condition. Int J Pediatr Otorhinolaryngol 79:2174-2177

3. Chiappini E, Ciarcià $\mathrm{M}$, Bortone $\mathrm{B}$, Doria $\mathrm{M}$, Becherucci $\mathrm{P}$, Marseglia GL, Motisi MA, de Martino M, Galli L, Licari A, de Masi S, Lubrano R, Bettinelli M, Vicini C, Felisati G, Villani A, Marchisio P, Italian Panel for the Management of Acute Otitis Media in Children (2019) Italian panel for the management of acute otitis media in children. Updated guidelines for the management of acute otitis media in children by the Italian Society of Pediatrics: Diagnosis. Pediatr Infect Dis J 38:S3-S9

4. Torretta S, Marchisio P (2017) Otitis media in children: a proposal for a new nosological classification. Int J Pediatr Otorhinolaryngol 93:174-175

5. Capaccio P, Torretta S, Marciante GA, Marchisio P, Forti S, Pignataro L (2016) Endoscopic adenoidectomy in children with otitis media with effusion and mild hearing loss. Clin Exp Otorhinolaryngol 9:33-38

6. Marchisio $\mathrm{P}$, Bortone $\mathrm{B}$, Ciarcià $\mathrm{M}$, Motisi MA, Torretta $\mathrm{S}$, Castelli Gattinara G, Picca M, di Mauro G, Bonino M, Mansi N, Varricchio A, Marseglia GL, Cardinale F, Villani A, Chiappini E (2019) Updated guidelines for the management of acute otitis media in children by the Italian Society of Pediatrics: Prevention. Pediatr Infect Dis J 38:S22-S36

7. Niemela N, Pihakari O, Pokka T, Uhari M (2000) Pacifier as a risk factor for acute otitis media: a randomized, controlled trial of parental counseling. Pediatrics 106:483-488

8. Torretta S, Marchisio P, Cappadona M, Baggi E, Pignataro L (2013) Habitual use of push and pull plastic bottle caps is more prevalent among children with recurrent acute otitis media. Int $\mathrm{J}$ Pediatr Otorhinolaryngol 77:1179-1182

9. Csákányi Z, Czinner A, Spangler J, Rogers T, Katona G (2012) Relationship of environmental tobacco smoke to otitis media (OM) in children. Int J Pediatr Otorhinolaryngol 76:989-993

10. Bowatte G, Tham R, Allen KJ, Tan DJ, Lau MXZ, Dai X, Lodge CJ (2015) Breastfeeding and childhood acute otitis media: a systematic review and meta-analysis. Acta Paediatr 104:85-95

11. Bowatte G, Tham R, Perret JL, Bloom M, Dong G, Waidyatillake N, Bui D, Morgan G, Jalaludin B, Lodge C, Dharmage S (2018) Air pollution and otitis media in children: a systematic review of literature. Int J Environ Res Public Health 15:257

12. Rosenfeld R, Bluestone C (1999) Evidence-based otitis media. BC Decker INC Hamilton, London

13. Rosenfeld RM, Kay D (2003) Natural history of untreated otitis media. Laryngoscope 113:1645-1657

14. Daly KA, Hoffman HJ, Kvaerner KJ, Kvestad E, Casselbrant ML, Homoe P, Rovers MM (2010) Epidemiology, natural history, and risk factors: panel report from the Ninth International Research Conference on Otitis Media. Int J Pediatr Otorhinolaryngol 74: 231-240

15. Alho OP, Läärä E, Oja H (1996) What is the natural history of recurrent acute otitis media in infancy? J Fam Pract 43:258-264

16. Prellner K, Foglé-Hansson M, Jørgensen F, Kalm O, Kamme C (1994) Prevention of recurrent acute otitis media in otitis-prone children by intermittent prophylaxis with penicillin. Acta Otolaryngol 114:182-187

17. Mandel EM, Casselbrant ML, Rockette HE et al (1996) Efficacy of antimicrobial prophylaxis for recurrent middle ear effusion. Pediatr Infect Dis J 15:1074-1082

Publisher's note Springer Nature remains neutral with regard to jurisdictional claims in published maps and institutional affiliations. 